\title{
Improving the Care of Elderly Adults Undergoing Surgery in Michigan
}

\author{
Kyle H. Sheetz, MS, Karen Guy, BS, James H. Allison, BA, Kara A. Barnhart, BS, \\ Scott R. Hawken, BS, Emily L. Hayden, BA, Jordan B. Starr, BS, Michael N. Terjimanian, MS, \\ Seth A. Waits, MD, Andrew J. Mullard, MS, Greta Krapohl, PhD, Amir A. Ghaferi, MD, MS, \\ Darrell A. Campbell, Jr, MD, and Michael J. Englesbe, MD
}

OBJECTIVES: To determine whether failure to rescue, as a driver of mortality, can be used to identify which hospitals attenuate the specific risks inherent to elderly adults undergoing surgery.

DESIGN: Retrospective cohort study.

SETTING: State-wide surgical collaborative in Michigan.

PARTICIPANTS: Older adults undergoing major general or vascular surgery between 2006 and 2011 ( $\mathrm{N}=24,216)$.

MEASUREMENTS: Thirty-four hospitals were ranked according to risk-adjusted 30-day mortality and grouped into tertiles. Within each tertile, rates of major complications and failure to rescue were calculated, stratifying outcomes according to age ( $<75$ vs $\geq 75)$. Next, differences in failure-to-rescue rates between age groups within each hospital were calculated.

RESULTS: Failure-to-rescue rates were more than two times as high in elderly adults as in younger individuals in each tertile of hospital mortality $(26.0 \%$ vs $10.3 \%$ at high-mortality hospitals, $P<.001)$. Within hospitals, the average difference in failure-to-rescue rates was $12.5 \%$. Nine centers performed better than expected, and three performed worse than expected, with the largest differences exceeding $25 \%$.

CONCLUSION: Although elderly adults experience higher failure-to-rescue rates, this does not account for hospitals' overall capacity to rescue individuals from complications. Comparing rates of younger and elderly adults within hospitals may identify centers where efforts toward complication rescue favor, or are customized for, elderly adults. These centers should be studied as part of the collaborative's effort to address the disparate outcomes

From the Academic Surgery Development Program, Department of Surgery, School of Medicine, University of Michigan, Ann Arbor, Michigan.

Address correspondence to Michael J. Englesbe, Department of Surgery, University of Michigan Medical School, 2926A Taubman Center, 1500 East Medical Center Drive, Ann Arbor, MI 48109. E-mail: englesbe@med. umich.edu

DOI: $10.1111 /$ jgs. 12643 that elderly adults in Michigan experience. J Am Geriatr Soc 62:352-357, 2014.

Key words: geriatric surgery; surgical quality assessment

$\mathrm{H}$ ospitals vary in their ability to avoid morbidity and mortality after major surgery. This observation is magnified in elderly adults, in whom surgical mortality is exponentially higher than in younger individuals. ${ }^{1,2}$ Intermediate outcomes in elderly adults also indicate that the risk of mortality after major surgery extends beyond the immediate postoperative period. ${ }^{3}$ The future significance of this is unknown but is relevant given projections that indicate that an increasing proportion of major surgical procedures will be performed on elderly adults. ${ }^{4}$ Furthermore, surgeons, hospitals, and insurers have parallel interests in this matter given the direct link between surgical outcomes and healthcare costs. ${ }^{5}$

Despite this, it remains unclear how best to reduce surgical mortality in elderly adults. In the Michigan Surgical Quality Collaborative (MSQC), a partnership between Blue Cross and Blue Shield of Michigan and 52 Michigan hospitals, surgical mortality has decreased in younger individuals over the past 5 years, whereas mortality in elderly adults has remained relatively constant. Previous work has highlighted the relationship between complication management and mortality (failure to rescue) as a principal driver of variation in hospital mortality and a suitable surgical quality metric. ${ }^{6-8}$ Many factors, including access to resources, care coordination, and the attitudes and behaviors of caregivers, influence failure-to-rescue rates. ${ }^{9}$ It is unknown whether processes of care that promote failure to rescue in younger adults are similar to those in elderly adults.

Within this context, data from the MSQC were used to study the relationship between mortality, major 
complications, and failure to rescue in Michigan hospitals. Outcomes of elderly and younger adults in the same hospitals were compared as a means of identifying centers where care of elderly adults was significantly better or worse than expected.

\section{METHODS}

\section{Data Source and Study Population}

Data from the MSQC prospective clinical registry from 2006 through 2011 at 34 member hospitals were used. This project followed standard data definitions and collection protocols within the MSQC as previously described. ${ }^{10,11}$ Dedicated MSQC data-collection nurses collect all data at the hospital level. Rigorous training of staff and data audits ensures accuracy and reliability of registry data. Information on all available variables, including participant demographic characteristics, preoperative risk factors, laboratory values, perioperative factors, and 30-day postoperative morbidity and mortality, was collected for this analysis. Additional data were obtained on hospital characteristics from the American Hospital Association database.

\section{Outcomes}

The primary outcomes for this study were 30-day inhospital mortality, major complications, and failure to rescue. Information on specific in-hospital postoperative complications such as surgical site infection (superficial, deep, and organ space defined separately), deep venous thrombosis, urinary tract infection, acute renal failure, postoperative bleeding requiring transfusion, stroke, unplanned intubation, fascial dehiscence, prolonged mechanical ventilation for longer than 48 hours, myocardial infarction, pneumonia, pulmonary embolism, sepsis, vascular graft loss, and renal insufficiency was gathered. Major complications included all but the following postoperative eventsurinary tract infection, deep venous thrombosis, renal insufficiency, and superficial surgical site infection, as has been previously described. ${ }^{7}$ Hospitals' failure-to-rescue rates were determined according to the proportion of participants dying after at least one major complication.

\section{Statistical Analysis}

Demographic tables were constructed. Demographic, comorbidity, and operative characteristics of participants younger than 75 were compared with those of participants aged 75 and older using the Student $t$-test, chi-square test, and Fisher exact test where appropriate. This age cutpoint was chosen because the risk of adverse events after surgery tends to increase significantly for participants between ages 70 and 80. Surgical populations are also increasing in age, with individuals aged 75 and older having the greatest growth. ${ }^{4}$ Hospitals were then ranked according to riskadjusted mortality. The unadjusted relationship between mortality and participant age was evaluated using simple linear regression and Pearson correlations.

Risk-adjustment models were developed using backward stepwise logistic regression that included variables such as participant age, sex, race, body mass index, diabetes mellitus, smoking status, alcohol use, dyspnea, do-notresuscitate (DNR) status, preoperative functional status, chronic obstructive pulmonary disease, pneumonia, ascites, congestive heart failure, need for dialysis, hemiplegia, transient ischemic attack, disseminated cancer, corticosteroid use, bleeding disorders, chemotherapy, radiotherapy, preoperative sepsis, esophageal varices, prior myocardial infarction, angina pectoris, hypertension requiring medication, peripheral vascular disease, prior operations, American Society of Anesthesiologists class, operative duration (minutes), surgeon specialty, work relative value units, and the need for intraoperative transfusion. The final model included 15 variables with a $\mathrm{C}$ statistic of 0.825 . The model was well calibrated across deciles of risk (HosmerLemeshow $P=0.16$ ).

Hospitals were subsequently grouped into tertiles based on risk-adjusted mortality rates (low, middle, high). Rates of major complications and failure to rescue (i.e. mortality after a major complication) were then evaluated for each tertile of mortality. Results were stratified according to participant age $(<75$ vs $\geq 75)$ to compare outcomes of interest for different ages at the same hospitals. A secondary analysis that included hospital characteristics (total bed size, nurse-patient ratio, inpatient surgical case volume) was performed to assess the influence of structural factors on failure to rescue.

Differences in failure-to-rescue rates of participants younger than 75 and 75 and older within the same hospital-the failure to rescue "gap"-were then calculated. Given that mortality was higher in elderly adults, some differences in failure-to-rescue rates were expected. This analysis sought to determine whether hospitals perform similarly well or poorly within age strata. To test for significance, $95 \%$ confidence intervals were calculated for the difference in proportions (failure-to-rescue rates) between age groups. These intervals were then compared with the average of all hospitals to identify which hospitals performed better or worse than expected than the average difference of all centers.

All statistical analyses were performed using Stata version 12.1 (Stata Corp., College Station, TX). The University of Michigan Institutional Review Board approved this study.

\section{RESULTS}

\section{Participant Population}

Individuals who underwent elective major general or vascular surgery within an MSQC member hospital between 2006 and 2011 were identified $(\mathrm{n}=24,216)$. Individuals aged 75 and older $(n=6,473)$ were $27 \%$ of the total study population. Descriptive statistics comparing the demographic, comorbidity, and procedural characteristics of the age groups are outlined in Table 1. In general, elderly adults had a greater comorbid disease burden and were more likely to have low serum albumin and to be functionally dependent. Although statistically significant, differences in mean work relative value units as a measure of case complexity were not appreciably different between age groups. 
Table 1. Participant Demographics Comparing Younger and Elderly Participants

\begin{tabular}{|c|c|c|c|}
\hline Characteristic & $\begin{array}{c}<75 \\
\mathrm{n}=17,743 \\
(73.4 \%)\end{array}$ & $\begin{array}{c}\geq 75 \\
n=6,473 \\
(26.6 \%)\end{array}$ & $P$-Value \\
\hline Age, median & 60.7 & 81.2 & $<.001$ \\
\hline Male, \% & 54.7 & 46.2 & $<.001$ \\
\hline Nonwhite, \% & 18.8 & 24.9 & $<.001$ \\
\hline Body mass index, $\mathrm{kg} / \mathrm{m}^{2}$, mean & 28.5 & 26.1 & $<.001$ \\
\hline \multicolumn{4}{|l|}{ Clinical } \\
\hline Diabetes mellitus, $\%$ & 73.6 & 72.4 & .80 \\
\hline Smoking in past year, \% & 37.9 & 11.7 & $<.001$ \\
\hline Do not resuscitate, \% & 0.7 & 3.4 & $<.001$ \\
\hline $\begin{array}{l}\text { Chronic obstructive pulmonary } \\
\text { disease, } \%\end{array}$ & 10.3 & 14.5 & $<.001$ \\
\hline Dialysis, \% & 4.8 & 4.2 & .04 \\
\hline Disseminated cancer, \% & 4.6 & 4.3 & .24 \\
\hline Corticosteroid use, $\%$ & 5.1 & 4.0 & $<.001$ \\
\hline Congestive heart failure, \% & 1.8 & 4.1 & $<.001$ \\
\hline $\begin{array}{l}>10 \% \text { weight loss in } 6 \text { months } \\
\text { before surgery, } \%\end{array}$ & 5.1 & 5.4 & .42 \\
\hline Albumin $<3.5 \mathrm{~g} / \mathrm{dL}, \%$ & 28.7 & 39.7 & $<.001$ \\
\hline $\begin{array}{l}\text { Independent functional } \\
\text { status, \% }\end{array}$ & 88.1 & 77.1 & $<.001$ \\
\hline $\begin{array}{l}\text { Hypertension requiring } \\
\text { medication, \% }\end{array}$ & 60.1 & 81.2 & $<.001$ \\
\hline Acute renal failure, \% & 0.9 & 1.1 & .19 \\
\hline $\begin{array}{l}\text { American Society of } \\
\text { Anesthesiologists class } \\
1 \text { or } 2, \%\end{array}$ & 31.5 & 14.2 & $<.001$ \\
\hline $\begin{array}{l}\text { Work relative value units, } \\
\text { mean }\end{array}$ & 24.3 & 23.3 & $<.001$ \\
\hline
\end{tabular}

\section{Mortality and Major Complications}

The relationship between unadjusted mortality and age was first evaluated (Figure 1). Although there is an overall correlation between age and postoperative mortality $(\rho=0.912)$, the relationship becomes nonlinear between ages 70 and 80 . Overall risk-adjusted mortality for hospitals ranged from $2.8 \%$ to $7.2 \%$. Average risk-adjusted mortality for participants younger than 75 was $2.2 \%$ at low-mortality hospitals, $3.1 \%$ at middle-mortality hospitals, and $3.2 \%$ at high-mortality hospitals $(P=.005$ low vs high) (Figure 2). Risk-adjusted mortality was higher and more variable for participants aged 75 and older at low-, middle-, and high-mortality hospitals $(6.0 \%, 9.8 \%$, and $10.3 \%, P<.001$ low vs high). Figure 2 shows major complication and failure-to-rescue rates for tertiles of hospital mortality. Although major complication rates were, in general, higher in participants aged 75 and older, there was no association between complication rates and mortality in either age group. For participants younger than 75 , major complication rates at low-, middle-, and highmortality hospitals were $23.2 \%, 26.8 \%$, and $21.8 \%$, respectively $(P=.08)$. In individuals aged 75 and older, major complication rates at low-, middle-, and high-mortality hospitals were $28.4 \%, 31.9 \%$, and $28.1 \%$, respectively $(P=.85)$.

\section{Failure to Rescue}

In contrast to major complication rates, failure-to-rescue rates increased in a stepwise fashion over tertiles of hospital mortality for both age groups (Figure 2). Failure-torescue rates in participants younger than 75 were $7.1 \%$, $9.3 \%$, and $10.3 \%$ in low-, middle-, and high-mortality hospitals, respectively ( $P=.007$ low vs high). A similar trend was observed in participants aged 75 and older across tertiles of hospital mortality $(15.4 \%, 22.5 \%$, and $26.0 \%, P<.001$ low vs high). Failure-to-rescue rates were also significantly different between age groups at each tertile of hospital mortality $(P<.001$ for each). A subset analysis was performed, excluding individuals with preoperative DNR orders, and similar results were obtained. It was also found that bed size of more than 200 beds (odds ratio $[\mathrm{OR}]=0.81,95 \%$ confidence interval $[\mathrm{CI}]=0.68-$ $0.97)$ had a significant influence on failure-to-rescue rates. This effect was not found in participants younger than 75 but was found in elderly adults $(\mathrm{OR}=0.75,95 \%$ $\mathrm{CI}=0.58-0.97)$. Higher nurse-patient ratio $(\mathrm{OR}=0.99$, $95 \% \mathrm{CI}=0.98-1.00)$ and inpatient surgical volume $(\mathrm{OR}=0.95,95 \% \mathrm{CI}=0.80-1.14)$ did not influence failure to rescue. After accounting for hospital characteristics, no differences were found from the individual-level analysis.

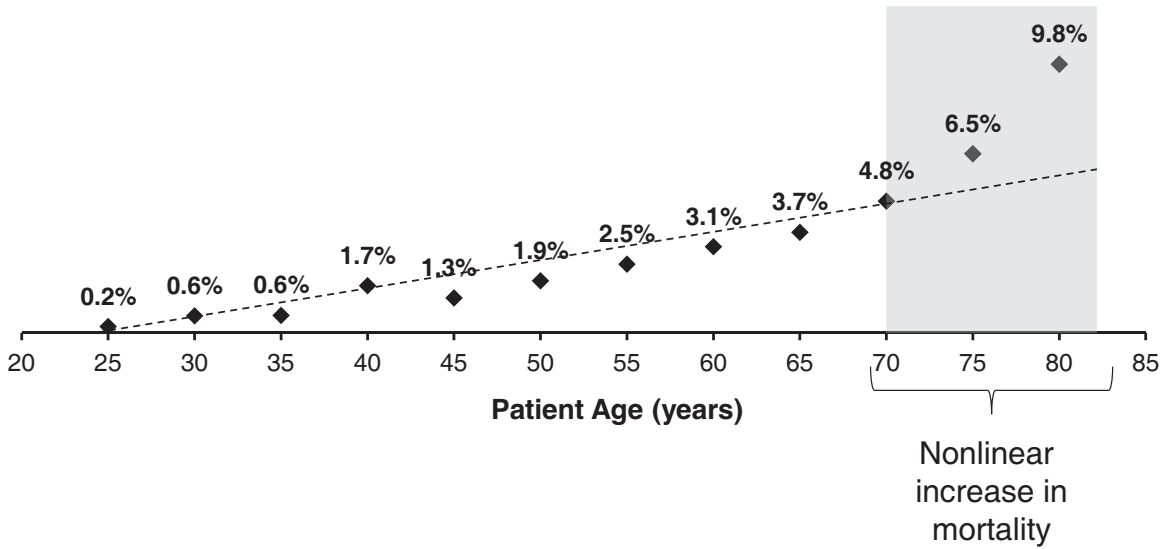

Figure 1. Scatterplot of unadjusted 30-day mortality over participant age. Mortality increases in a linear fashion (trend line) until approximately age 70, at which point rates increase in a larger, nonlinear pattern (blue box). 


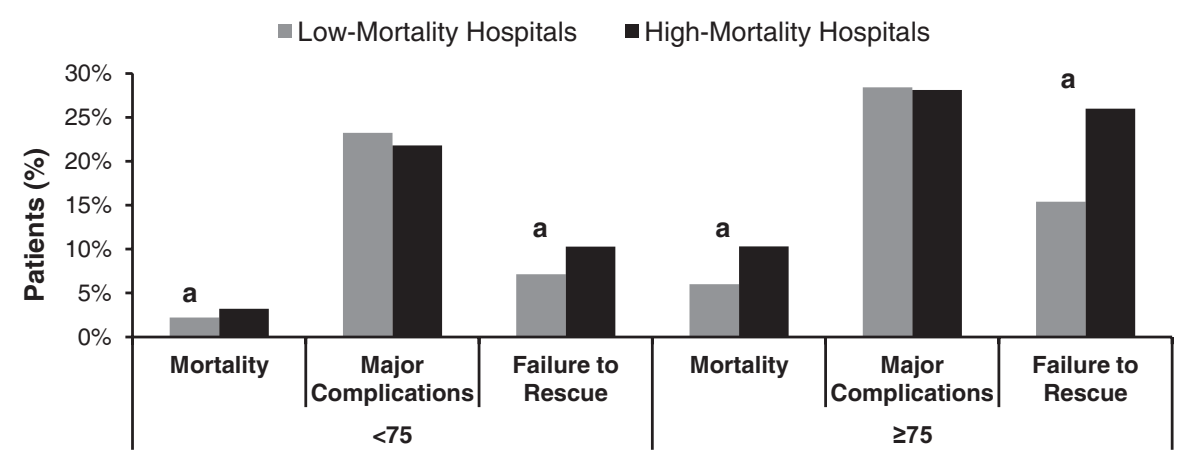

Figure 2. Mortality and major complication and failure-to-rescue rates for younger $(<75)$ and elderly $(\geq 75)$ participants stratified according to tertile of hospital mortality (low- and high-mortality tertiles shown). Major complication rates are not significantly different between tertiles of hospital mortality. Failure to rescue correlates with mortality and is significantly higher in elderly adults ( ${ }^{a}$ significant differences between high- and low-mortality hospitals, $P<.01$ ).

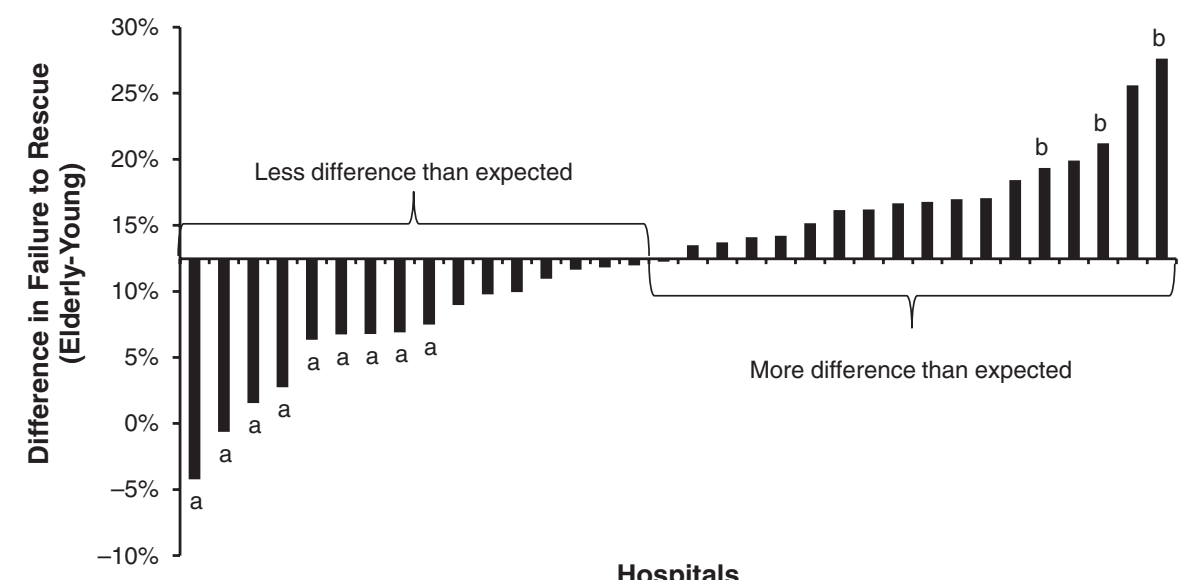

Figure 3. Differences in failure-to-rescue rates between younger $(<75)$ and elderly $(\geq 75)$ adults stratified according to hospital. Hospitals on the left have less difference ("gap") in rates, whereas those on the right have a greater difference. Hospitals are identified if their rate difference is significantly better $\left({ }^{a}\right)$ or worse $\left({ }^{b}\right)$ than the overall average $(95 \%$ confidence interval).

\section{The Failure-to-Rescue "Gap"}

The mean difference in failure-to-rescue rates between age groups was $12.5 \%$ for all hospitals. Figure 3 shows the magnitude of differences in failure-to-rescue rates between age groups in reference to the overall mean. There were nine centers with smaller gaps than expected (the magnitude of difference between age groups was less than the overall average). In contrast, three centers performed significantly worse than expected, with large differences in failure-to-rescue rates between age groups. In other words, there were substantial differences in failure-to-rescue rates between individuals younger than 75 and those aged 75 and older at these three hospitals.

\section{DISCUSSION}

This study addresses a growing body of literature suggesting the importance of complication rescue for reducing mortality in elderly adults undergoing surgery. It highlights not only that elderly adults are more susceptible to major complications, but also that the variation in mortality and failure to rescue is more significant than in younger individuals. Thirty-day mortality after elective major general or vascular surgery was higher and more variable in elderly adults than in younger individuals. Although rates of major complications were similar across tertiles of hospital mortality, failure-to-rescue rates increased in a stepwise manner from low- to high-mortality hospitals in both age groups. Significant variation was also observed in the differences in failure-to-rescue rates between age groups at the same hospital. The failure-to-rescue "gap" accounts for a hospital's baseline performance (failure to rescue in younger individuals) and identifies centers where outcomes deviate more or less than expected in a more-at-risk elderly population. The collaborative plans to visit and engage these high-performing sites, using them as mentors for an implementation program focusing on surgical care of older adults.

Numerous studies have highlighted the disproportionately poor outcomes that elderly adults undergoing major surgery experience. ${ }^{1,12,13}$ Understanding the mechanisms that drive these outcomes is less clear. Preoperative optimization of comorbid conditions and improvement of overall functional status has become a focus of nationwide collaborative efforts between surgeons and geriatric specialists. ${ }^{14}$ 
Although this is important, there are data to suggest that these efforts may be of questionable benefit in the way of morbidity and mortality reduction. ${ }^{15,16}$ When focusing efforts on the postoperative period, there is strong evidence to suggest the importance of complication management rather than prevention (failure to rescue). ${ }^{6,7,17}$ Further study has identified several hospital characteristics associated with low failure-to-rescue rates, including overall case volume, intensive care unit availability, hospital bed size, and nurse-patient ratios. ${ }^{9}$ No studies have explicitly studied the relationship between complication rescue and mortality in elderly and younger individuals undergoing elective major surgery as a metric for hospital quality.

Similarly, many broad-based quality improvement efforts such as the MSQC have included individuals of all ages, allowing for broadly generalizable results, ${ }^{11}$ but this approach neglects adverse outcomes arising in specific vulnerable populations, such as elderly adults. Targeted quality improvement efforts in elderly adults are relevant considerations for surgeons and hospitals as this population increases in prevalence over the next 20 years. ${ }^{18}$ It has been the practice of the collaborative to disseminate the best practices of high-quality centers statewide. These data suggest that, although elderly adults have worse outcomes, some hospitals may be attenuating the inherent risk of treating these vulnerable individuals and the differences in mortality that result. These "positive deviants" may represent an important focus in addressing differences in rescue between individuals of different ages. ${ }^{19}$ Caregivers' abilities to rescue individuals from complications are related to hospital resources discussed above, but addressing these factors (e.g., bed size) may not be possible for hospitals. This places emphasis on understanding how hospitals care for elderly adults with available resources-a logical next step for the collaborative given the results of this analysis. Furthermore, age-specific performance should be considered as a more-robust measure of surgical quality for individual hospitals than aggregate performance in individuals of all ages.

As with any retrospective cohort study, their vulnerability to confounders limit these findings. Although the MSQC data set is considered the criterion standard in registry-level surgical data, determining the importance of patient-, surgeon-, and hospital-level factors is difficult. There are also potential confounding variables that are not measured. It was not possible to obtain information on participants' insurance or socioeconomic status. With regard to surgeon level factors, previous experience with these data has indicated that small sample sizes plague provider-specific performance measurement. ${ }^{10}$ Hospitallevel factors were purposefully not controlled for. Considering that this study specifically aimed to inform quality-improvement efforts, controlling for hospital-level factors could obscure significant differences that will instead be carefully considered as hospitals' practices are assessed. Last, 34 hospitals in Michigan, all of which are participating in a longitudinal quality-improvement collaborative, are being studied. Whether these findings are generalizable to hospitals outside of Michigan is uncertain.

Failure to rescue is a fundamental driver of hospital variation in mortality after elective major general or vascular surgery. Differences in failure to rescue between young and elderly adults may underlie differential practice in the management of these high-risk individuals undergoing surgery. Differences in outcomes between age groups within the same hospital may be used as an additional quality metric. The results of this study encourage further investigation into the practices of Michigan hospitals. In identifying the factors that affect the outcomes of elderly adults, special consideration should be given to characteristics that contribute to effective, timely, and age-appropriate complication management.

\section{ACKNOWLEDGMENTS}

Conflict of Interest: The editor in chief has reviewed the conflict of interest checklist provided by the authors and has determined that the authors have no financial or any other kind of personal conflicts with this paper.

Author Contributions: Sheetz K. H., Guy K., Waits S. A., Krapohl G., Ghaferi A. A., Campbell D. A., Englesbe M. J.: study concept and design. Sheetz K. H., Guy K., Allison J. H., Barnhart K. A., Hawken S. R., Hayden E. L., Starr J. B., Terjimanian M. N., Waits S. A., Mullard A. J., Krapohl G.: acquisition of data. Sheetz K. H., Guy K., Allison J. H., Barnhart K, A., Hawken S. R., Hayden E. L., Starr J. B., Terjimanian M. N., Mullard A. J., Krapohl G., Ghaferi A. A., Campbell D. A., Englesbe M. J.: analysis and interpretation. Sheetz K. H., Guy K., Waits S. A., Krapohl G., Ghaferi A. A., Campbell D. A., Englesbe M. J.: preparation of manuscript.

Sponsor's Role: None.

\section{REFERENCES}

1. Finlayson EV, Birkmeyer JD. Operative mortality with elective surgery in older adults. Eff Clinl Pract 2001;4:172-177.

2. Duron J-J, Duron E, Dugue T et al. Risk factors for mortality in major digestive surgery in the elderly: A multicenter prospective study. Ann Surg 2011;254:375-382.

3. Kwok AC, Semel ME, Lipsitz SR et al. The intensity and variation of surgical care at the end of life: A retrospective cohort study. Lancet 2011;378:1408-1413.

4. Etzioni DA, Liu JH, Maggard MA, et al. The aging population and its impact on the surgery workforce. Ann Surg 2003;238:170-177.

5. Birkmeyer JD, Gust C, Dimick JB et al. Hospital quality and the cost of inpatient surgery in the United States. Ann Surg 2012;255:1-5.

6. Ghaferi AA. Complications, failure to rescue, and mortality with major inpatient surgery in Medicare patients. Ann Surg 2009;250: 1029-1034.

7. Ghaferi AA, Birkmeyer JD, Dimick JB. Variation in hospital mortality associated with inpatient surgery. N Engl J Med 2009;361:1368-1375.

8. Silber JH, Williams SV, Krakauer H et al. Hospital and patient characteristics associated with death after surgery. A study of adverse occurrence and failure to rescue. Med Care 1992;30:615-629.

9. Ghaferi AA, Osborne NH, Birkmeyer JD et al. Hospital characteristics associated with failure to rescue from complications after pancreatectomy. J Am Coll Surgeons 2010;211:325-330.

10. Campbell DA Jr, Englesbe MJ, Kubus JJ et al. Accelerating the pace of surgical quality improvement: The power of hospital collaboration. Arch Surg 2010;145:985-991.

11. Englesbe MJ, Brooks L, Kubus J et al. A statewide assessment of surgical site infection following colectomy: The role of oral antibiotics. Ann Surg 2010;252:514-519; discussion 519-520.

12. Turrentine FE, Wang H, Simpson VB et al. Surgical risk factors, morbidity, and mortality in elderly patients. J Am Coll Surg 2006;203: $865-877$.

13. Ingraham AM, Cohen ME, Raval MV et al. Variation in quality of care after emergency general surgery procedures in the elderly. J Am Coll Surg 2011;212:1039-1048 
14. Chow BK, Rosenthal C, Esnaola R. ACS-NSQIP/AGS Best Practice Guidelines: Optimal Preoperative Assessment of the Geriatric Surgical Patient. Chicago, IL: American College of Surgeons, 2012.

15. Ramos M. Relationship of perioperative hyperglycemia and postoperative infections in patients who undergo general and vascular surgery. Ann Surg 2008;248:585-591.

16. Fleisher LA, Eagle KA. Clinical practice. Lowering cardiac risk in noncardiac surgery. N Engl J Med 2001;345:1677-1682.
17. Ghaferi AA. Hospital volume and failure to rescue with high-risk surgery. Med Care 2011;49:1076-1081.

18. Hobbs F, Stoops N U.S. Census Bureau, Census 2000 Special Reports, Series CENSR-4, Demographic Trends in the 20th Century. Washington, DC: U.S. Administration on Aging, 2008.

19. Bradley EH, Curry LA, Ramanadhan S et al. Research in action: Using positive deviance to improve quality of health care. Implement Sci 2009;4:25. 